\title{
Somatic PIK3CA mutations are present in multiple tissues of facial infiltrating lipomatosis
}

\author{
Javier A. Couto ${ }^{1}$, Dennis J. Konczyk ${ }^{1}$, Matthew P. Vivero ${ }^{1}$, Harry P.W. Kozakewich ${ }^{2}$, Joseph Upton ${ }^{1}$, Xi Fu', Bonnie L. Padwa ${ }^{1}$,
} John B. Mulliken ${ }^{1}$, Matthew L. Warman ${ }^{3}$ and Arin K. Greene ${ }^{1}$

BACKGROUND: Facial infiltrating lipomatosis (FIL) is a congenital disorder that causes overgrowth of one side of the face. The purpose of this study was to determine whether PIK3CA mutations are present in tissues outside of the subcutaneous adipose.

METHODS: FIL tissues from three patients were dissected to enrich for cells from skin, subcutaneous tissue, orbicularis oris muscle, buccal fat, zygomatic bone, and mucosal neuroma. Endothelial cells within the affected tissue also were enriched using CD31 microbeads. Laser capture microdissection on formalin-fixed paraffin-embedded histologic sections was performed to collect specific cell types. DNA was extracted from each tissue and cell type, and measured for the abundance of mutant PIK3CA alleles using droplet digital PCR. RESULTS: We detected mutant PIK3CA alleles in every tissue and cell type tested from each overgrown face; frequencies ranged from 1.5 to $53 \%$. There were fewer mutant endothelial cells compared with nonendothelial cells, and the stromal cell compartment had the highest frequency of mutant cells in each tissue.

CONCLUSIONS: PIK3CA mutations are not restricted to a single tissue or cell type in FIL. Overgrowth in this condition is likely due to the mutation arising in a cell that contributes to several different facial structures during embryogenesis.

$\mathbf{F}$ acial infiltrating lipomatosis (FIL) is a nonhereditary disorder characterized by hemifacial overgrowth with significant subcutaneous adipose hypertrophy. Patients have enlargement of one side of the face and also can have mucosal neuromas, hemimacroglossia, bony hypertrophy, and premature dental eruption $(1,2)$. We previously reported that subcutaneous adipose tissue from the affected areas of patients with FIL contain somatic activating mutations in PIK3CA (E453K, E542K, H1047R, and H1047L), a gene encoding the catalytic component of phosphatidylinositol 3-kinase (3). Thus, FIL is a part of the PIK3CA-related overgrowth spectrum (PROS), along with other hypertrophy conditions caused by PIK3CA mutations (4). Although FIL typically is an isolated disease, patients with CLOVES (congential lipomatous overgrowth, vascular malformations, epidermal nevi, and scoliosis/skeletal/spinal anomalies) can have FIL as a component of their syndrome (5).

Because our previous study only tested for PIK3CA mutations in subcutaneous fat, it is unknown whether mutant cells reside exclusively in the adipose tissue or are more widely distributed. The former hypothesis would suggest that facial overgrowth is primarily driven by paracrine signaling from fat cells, whereas the latter would determine that hypertrophy is due to the presence of mutant cells in the overgrown tissues. To address these two potential mechanisms for overgrowth, we searched for PIK3CA mutations in different cell and tissue types that were excised from patients with FIL during a clinically indicated procedure.

\section{METHODS}

Patient Ascertainment and Sample Collection

After approval by the Committee on Clinical Investigation at Boston Children's Hospital and acquiring written consent, tissue was collected from three patients with FIL who underwent subtotal resection. Participant 1 was a 3-year-old male (Figure 1a), participant 2 was an 8-year-old female, and participant 3 was a 15-year-old female; all subjects had isolated FIL and had no other comorbidities or features associated with another PROS condition (e.g., CLOVES syndrome) (Table 1). Samples collected from the study participants included skin and subcutaneous tissue, orbicularis oris muscle, buccal fat, zygomatic bone, and/or oral mucosal neuromas. Non-affected tissue (white blood cell DNA) was acquired from two of the patients. To more precisely define cell types within specimens, some samples were formalin-fixed and paraffinembedded so that histologic sections could be microscopically examined and used for laser capture microdissection. Because subcutaneous adipose was known to contain the PIK3CA mutation and was the most abundant tissue collected, a portion was used to enrich for vascular endothelial cells in all three participants.

Participant 1 was a 3 -year-old male who was noted to have rightsided facial overgrowth at birth. He did not have any other medical problems, family history of overgrowth, or medications. On physical examination, he had enlargement of the right face, as well as mucosal neuromas, macrodontia, and hemimacroglossia (Figure 1a). These features were pathognomonic for FIL. He underwent magnetic resonance imaging which confirmed the diagnosis-significant adipose hypertrophy of the subcutaneous tissue with overgrowth of all structures of one side of the face. The patient did not have any features of a syndrome associated with PROS (e.g., epidermal nevus,

\footnotetext{
'Department of Plastic \& Oral Surgery, Boston Children's Hospital, Boston, Massachusetts; ${ }^{2}$ Department of Pathology, Boston Children's Hospital, Boston, Massachusetts; ${ }^{3}$ Department of Orthopaedic Surgery, Boston Children's Hospital, Boston, Massachusetts. Correspondence: Arin K. Greene (arin.greene@childrens.harvard.edu) 
a

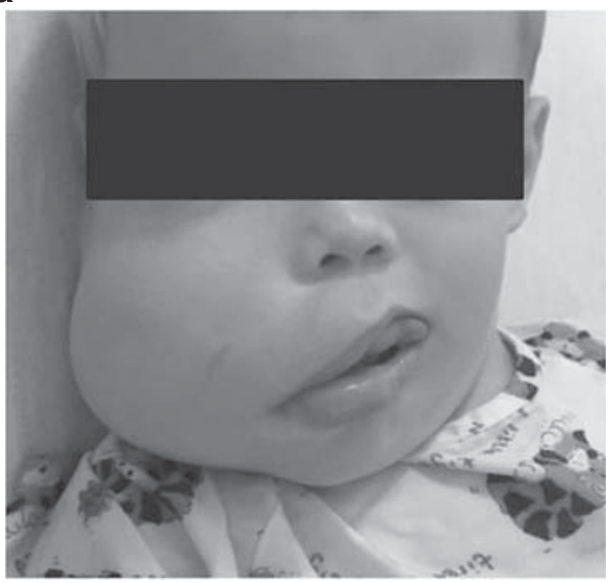

b

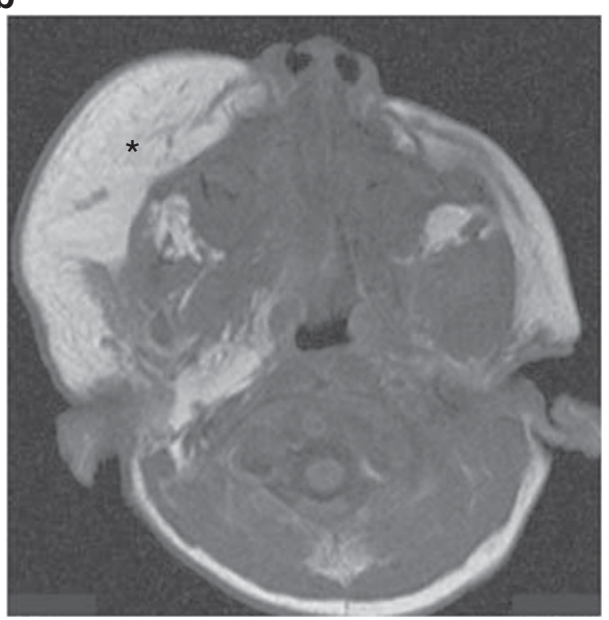

\section{C}

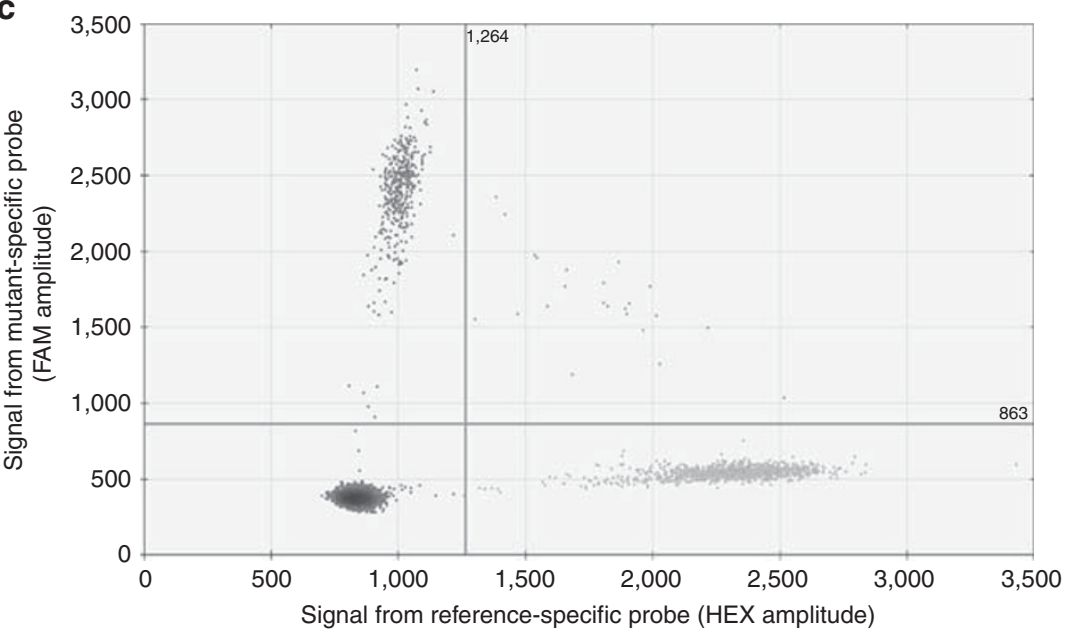

d

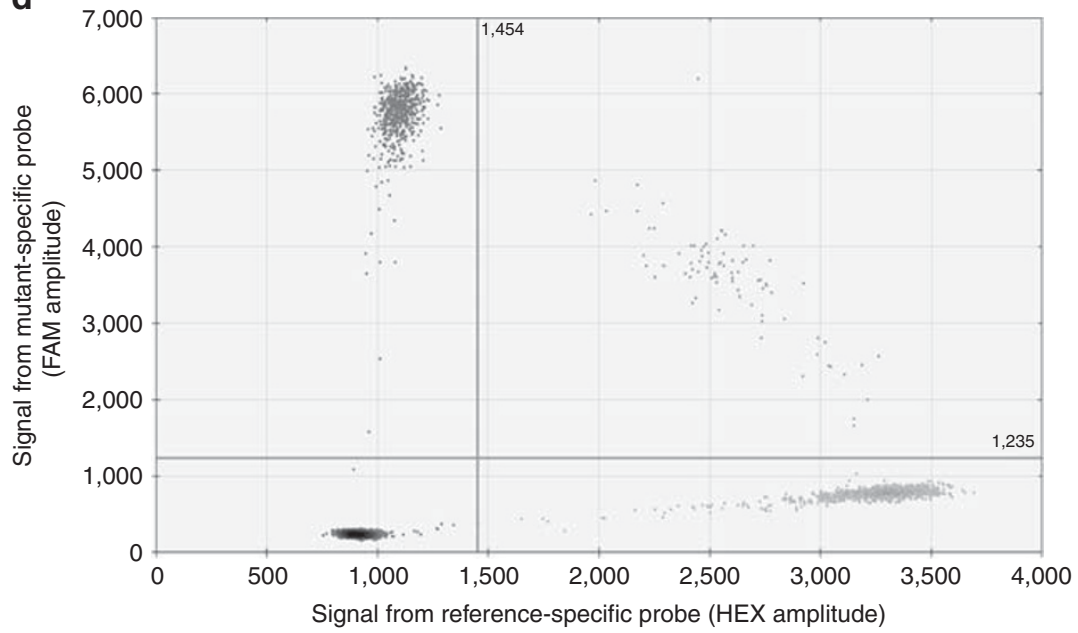

Figure 1. Clinical images and droplet digital PCR (ddPCR) data for participant 1. (a) Three-year-old male before a debulking procedure. (b) Magnetic resonance imaging shows increased subcutaneous thickening $\left(^{*}\right)$. (c, d) ddPCR assay results for DNA extracted from mucosal neuroma (c) and from zygoma (d). Droplets containing amplimers representing mutant alleles are FAM-positive (left upper quadrant). Droplets containing amplimers representing wild-type alleles are HEX-positive (right lower quadrant). Droplets with mutant and wild-type amplimers are located in the right upper quadrant. Droplets without amplimers are located in the left lower quadrant.

Table 1. Study cohort with FIL

\begin{tabular}{lccc}
\hline & Participant 1 & Participant 2 & Participant 3 \\
\hline PIK3CA mutation & p.H1047R & p.H1047R & p.H1047L \\
Age at operation (years) & 3 & 8 & 15 \\
Sex & Male & Female & Female \\
Side of facial overgrowth & Right & Left & Left \\
Hemifacial overgrowth & + & + & + \\
Mucosal neuromas & + & + & + \\
Macrodontia & + & + & + \\
Hemimacroglossia & + & + & + \\
Epidermal nevus & - & - & - \\
Hand or foot anomalies & - & - & - \\
Spinal anomalies & - & - & - \\
Brain anomalies & - & - & - \\
Vascular malformations & - & - & - \\
\hline
\end{tabular}

hand/foot anomalies, spinal malformations, brain anomalies, and vascular malformations).

Participant 2 was an 8-year-old female who had overgrowth of the left side of her face at birth. She did not have any other medical problems and was not taking medications. Family history was unremarkable. Physical examination showed features consistent with FIL: hemifacial overgrowth, mucosal neuromas, macrodontia, and hemimacroglossia. CT imaging confirmed adipose hypertrophy in the subcutaneous plane and overgrowth of all structures of the left face. She did not have features of any other PROS condition (e.g., epidermal nevus, hand/foot anomalies, spinal malformations, brain anomalies, and vascular malformations).

Participant 3 was a 15 -year-old female who was noted to have overgrowth of her left face at birth. She was otherwise healthy and there was no family history of overgrowth conditions. Physical examination was pathognomonic for FIL (hemifacial enlargement, mucosal neuromas, macrodontia, and hemimacroglossia). Magnetic resonance imaging showed subcutaneous adipose excess and overgrowth of all facial structures of the left face. She did not exhibit any features of other PROS conditions (e.g., epidermal nevus, hand/foot 


\section{Articles $\mid$ Couto et al.}

anomalies, spinal malformations, brain anomalies, and vascular malformations).

\section{Laser Capture Microdissection}

Five micron-thick formalin-fixed, paraffin-embedded tissue sections were obtained from the grossly dissected orbicularis oris muscle, buccal fat, mucosal neuroma, skin, and bone. Laser capture microdissection was used to enrich for specific cell types within these sections. From the orbicularis oris specimen, skeletal muscle cells, stromal cells, and adipocytes were collected. From the buccal fat, stromal cells and adipocytes were obtained. Osteocytes were collected from the bone. Mucosal neuroma-derived epithelial, stromal, and neural cells were isolated. From the skin, epidermis and dermis, adipocytes, and stromal cells were collected. Because tissue sections were photographed before and after laser microdissection, hematoxylin and eosin staining was done without a coverslip; water used as a surface film during photography caused a variable amount of eosin to leach out of the sections.

Laser-captured specimens were suspended in $30 \mu \mathrm{l}$ of phosphate buffered saline, to which $30 \mu \mathrm{l}$ of $25 \mathrm{~mm} \mathrm{NaOH}$ and $0.2 \mathrm{~mm}$ disodium EDTA, and $30 \mu \mathrm{l}$ of $40 \mathrm{~mm}$ Tris- $\mathrm{HCl}$ were sequentially added following the HotSHOT method (6). Two microliters of these HotSHOT-extracted template DNAs was then used to PCR-amplify the mutated region of PIK3CA, using previously described primers and PCR conditions (7). PCR product was quantified using a spectrophotometer (DS-11, DeNovix, Wilmington, DE, USA).

\section{Cell Sorting and Analysis}

Subcutaneous adipose tissue was washed in PBS to remove blood cell contaminants, digested with collagenase A $(2.5 \mathrm{mg} / \mathrm{ml})$ (Roche, Basal, Switzerland) for $1 \mathrm{~h}$ at $37^{\circ} \mathrm{C}$, then filtered through a $100 \mu \mathrm{m}$ strainer to produce a single-cell suspension. Cells were placed on fibronectin-coated $\left(1 \mu \mathrm{g} / \mathrm{cm}^{2}\right)$ tissue culture plates (Genesee Scientific, San Diego, CA, USA) in endothelial growth medium-2 (EGM-2, Lonza, Basal, Switzerland) supplemented with $10 \%$ fetal bovine serum (FBS, Gibco, Gaithersburg, MD, USA; Life Technologies). After 5-7 days of expansion, cells were fractionated into two populations (endothelial and nonendothelial) using anti-human CD31 (endothelial cell marker) magnetic beads (DynaBeads, Life Technologies, Carlsbad, CA, USA) (8). DNA was extracted from each cell population using the QIAamp DNA Mini kit following the manufacturer's instructions (Qiagen, Hilden, Germany).

\section{Droplet Digital PCR (ddPCR)-Based Mutational Analysis}

PCR primers, fluorescent probes, and methods for detecting wildtype and mutant PIK3CA alleles have been previously described (7).
For genomic DNA that was extracted from tissue or cells, we used $30 \mathrm{ng}$ of template DNA, corresponding to $\sim 4000$ cells, in each ddPCR reaction. For PCR amplimers that were produced using laser capture microdissected DNA as template, we used $0.3 \mathrm{fg}$ of DNA, corresponding to 3000 DNA molecules, in each ddPCR reaction. ddPCR was performed using a QX200 Droplet Generator, QX200 Droplet Reader, and QuantaSoft software (Bio-Rad, Hercules, CA, USA).

\section{RESULTS}

Using ddPCR, we detected either a p.H1047R or p.H1047L missense mutant PIK3CA allele in every tissue and cell type examined from each overgrown face; a mutation was not present in unaffected tissue (white blood cell DNA) (Table 2, Figure 1). To exclude the possibility that mutant alleles in nonadipose tissues were derived from microscopic adipocyte infiltration, we examined stained histologic sections of the zygomatic bone, skin, orbicularis oris muscle, and mucosal neuroma specimens from which we extracted DNA for ddPCR. We observed only sparsely distributed adipocytes in the orbicularis oris muscle specimen. Therefore, we performed ddPCR on DNA that was recovered from the muscle, adipocyte, and stromal cell components of this specimen by laser capture microdissection (Figure 2). In the orbicularis oris specimen, the myocyte-enriched component contained $24 \%$ mutant alleles, whereas the adipocyte and stromal components contained 25 and $41 \%$ mutant alleles, respectively (Table 3 ). Results consistent with involvement of multiple cell types also were obtained when the buccal fat, mucosal neuroma, and skin were studied (Table 3). The stromal cell compartment contained the highest frequency of mutant cells in each section.

Because it is challenging to laser capture vascular vs. nonvascular cells in order to determine whether endothelial cells were enriched for the PIK3CA mutation, we used an affinity bead-based method to enrich for $\mathrm{CD}^{+} 1^{+}$vascular endothelial cells in each participant's subcutaneous tissue specimen. In contrast to the nonendothelial cell compartment, which had high levels of mutant cells, the endothelial cell compartment had low levels (Table 2).

Table 2. PIK3CA mutant allele frequencies (\%) in tissues, endothelial cells, and nonendothelial cells

\begin{tabular}{|c|c|c|c|c|c|c|c|c|c|}
\hline Participant & Mutation & Skin & Subcutis & Muscle & Buccal fat & Bone & Mucosal Neuroma & $\mathrm{CD} 31^{+}$ & $\mathrm{CD}^{2} 1^{-}$ \\
\hline 1 & p.H1047R & 11.4 & 20.1 & 28.2 & 29.0 & 24.6 & 12.8 & - & 49.2 \\
\hline 2 & p.H1047R & 5.5 & 17.6 & 20.7 & 23.2 & - & - & 5.2 & 39.4 \\
\hline
\end{tabular}

(-) tissue not available for mutational analysis

Figure 2. Laser capture microdissection of tissue specimens from participant 1. (a) (Left) Orbicularis oris shows striated muscle $(\rightarrow)$, stroma $(\Delta)$, and adipocytes $(*)$. (Right) Post-capture image illustrates microdissection of myocyte-enriched areas (1) (microdissection of adipocytes and stromal tissue also was performed, data not shown) (original magnification $\times 40$ ). (b) (Left) Buccal fat lobules composed of adipocytes (*) surrounded by fibrous septa $(\rightarrow)$. (Right) Tissue section after laser capture of adipocytes (2) and septa (3) for DNA extraction (original magnification $\times 40)$. (c) (Left) Mucosal neuroma comprised of the epithelium (\{\}), stroma $(\Delta)$, and an enlarged nerve $(\mathbf{\Delta})$. (Right) Tissue section after laser capture of epithelium (4), stroma $(5)$, and nerve (6) (original magnification $\times 40)$. (d) (Left) Skin and subcutaneous tissue section show the epidermis and dermis (a), adipocytes $(*)$ and fibrous septa $(\rightarrow)$ of the subcutaneous tissue $(\beta)$. (Right) Post-capture of the epidermis and dermis (7), adipocytes (8), and stromal tissue (9) (original magnification $\times 20$ ). (hematoxylin and eosin staining). 


\section{PIK3CA mutations in multiple FIL tissues 1 Articles}
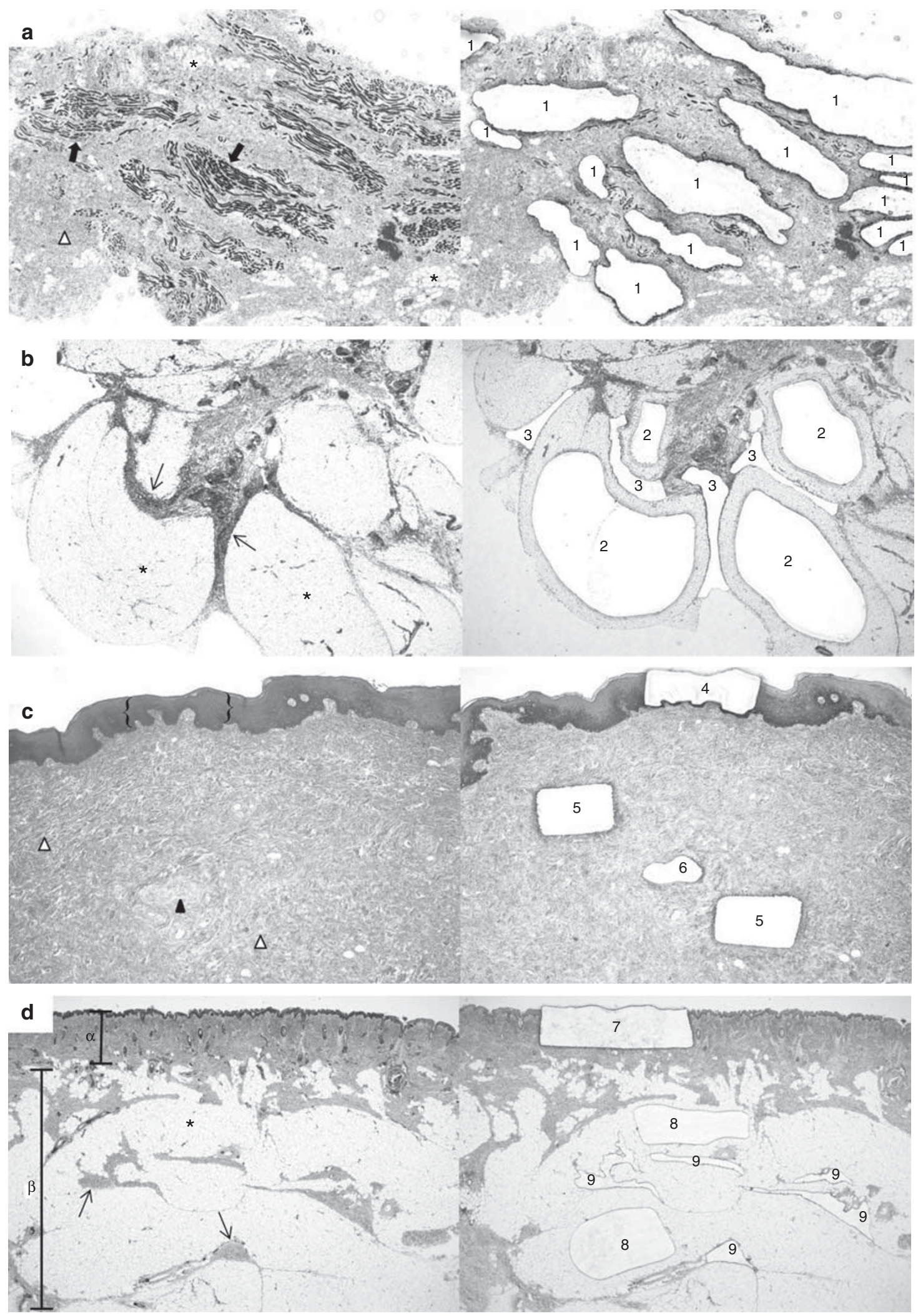

DISCUSSION

Surgical resection is the only therapeutic option to treat FIL. To develop pharmacological interventions, previously we searched for the underlying cause of FIL and found that the affected tissue contained somatic gain-of-function mutations in PIK3CA (E453K, E542K, H1047R, and H1047L) (3). These same somatic mosaic mutations have been identified in cancers, vascular malformations, and other disorders with overgrowth $(4,7,9)$. In 
Table 3. PIK3CA mutant allele frequencies (\%) in microdissected tissue

\begin{tabular}{lcc}
\hline Specimen & Label in Figure 2 & Mutant allelic frequency \\
\hline Bone cells & & 17.1 \\
Buccal fat & 2 & \\
$\quad$ Adipocytes & 3 & 23.2 \\
$\quad$ Stromal cells & & 52.8 \\
Muscle & 1 & \\
$\quad$ Skeletal muscle cells & $*$ & 23.7 \\
Adipocytes & $\Delta$ & 25.4 \\
Stromal cells & & 40.7 \\
Oral mucosa & & \\
Epithelium & 4 & 2.4 \\
Nerves & 6 & 32.7 \\
Stromal cells & 5 & 41.3 \\
Skin & 7 & 17.3 \\
Subcutis & & \\
Adipocytes & 8 & 31.9 \\
Stromal cells & 9 & \\
\hline$*$ * adipocytes in muscle specimen & 4 stroma in muscle specimen.
\end{tabular}

the present study, we sought to determine whether overgrowth in patients with FIL was caused by a somatic mutation in one tissue type (subcutaneous adipose) that exerts paracrine effects on neighboring structures (10)or by the presence of mutant cells in each of a patient's overgrown tissues (11).

We found that all tissues on the side of the face affected with FIL contained PIK3CA (H1047R, H1047L) mutations. A similar paradigm has been found in another PROS condition (CLOVES), where a patient who had an amputated limb was noted to have PIK3CA mutations in multiple tissues of the overgrown extremity (12). FIL exhibited the highest mutant allele frequency in the stroma, potentially because fibroblasts might have a greater mutant allele frequency compared with other cell types. Another possibility is that mutant progenitor cells reside in the stroma that can differentiate into parenchymal cells.

FIL had been hypothesized to result from a vascular abnormality because patients often have increased cutaneous vascularity exemplified as a stain or blush $(1,2)$. We recently found that endothelial cells from patients with a different overgrowth syndrome, capillary malformation, are enriched for GNAQ mutations (13). We did not find the same enrichment of PIK3CA mutant endothelial cells in patients with FIL. Therefore, overgrowth in patients with FIL is unlikely to be caused by an underlying vascular abnormality. Instead, our data indicate that FIL results from a mutation in a multipotent progenitor cell that contributes to mesodermal derivatives (e.g., stroma, adipose, muscle, and bone) as well as to ectodermal structures (e.g., nerves) $(14,15)$. If we had found that only the subcutaneous adipose tissue contained PIK3CA mutations, we would postulate that these adipocytes have nonautonamous effects on other facial cells that cause overgrowth. A dynamic paracrine interaction between cell types might be more favorable to develop pharmacotherapy for the disease; for example, inhibitors of paracrine signaling by subcutaneous adipose might prevent secondary overgrowth of other structures. Instead, all components of the face contain mutations that likely occurred during embryogenesis, suggesting that the condition is a structural anomaly. Mice that harbor conditional alleles in Pik3ca, which can be activating in a celltype- and tissue-specific manner, likely will be useful in further elucidating the cause of FIL.

\section{ACKNOWLEDGMENTS}

We thank Lois E. Smith and Zhongjie Fu from the Department of Ophthalmology, Boston Children's Hospital, for letting us use their laser capture microdissection system. This work was supported by the Translational Research Program, Boston Children's Hospital (A.K.G.), and NIH grants R21-HD082606 (A.K.G.), R21-HD081004 (A.K.G), and R01-AR064231 (M.L.W.).

Disclosure: The authors declare no conflict of interest.

\section{REFERENCES}

1. Slavin SA, Baker DC, McCarthy JG, Mufarrij A. Congenital infiltrating lipomatosis of the face: clinicopathologic evaluation and treatment. Plast Reconstr Surg 1983;72:158-64.

2. Padwa BL, Mulliken JB. Facial infiltrating lipomatosis. Plast Reconstr Surg 2001;108:1544-54.

3. Maclellan RA, Luks VL, Vivero MP, et al. PIK3CA activating mutations in facial infiltrating lipomatosis. Plast Reconstr Surg 2014;133:12e-9e.

4. Keppler-Noreuil KM, Rios JJ, Parker VE, et al. PIK3CA-related overgrowth spectrum (PROS): diagnostic and testing eligibility criteria, differential diagnosis, and evaluation. Am J Med Genet A 2015;167A:287-95.

5. Fernandez-Pineda I, Fajardo M, Chaudry G, Alomari AI. Perinatal clinical and imaging features of CLOVES syndrome. Pediatr Radiol 2010;40:1436-9.

6. Truett GE, Heeger P, Mynatt RL, Truett AA, Walker JA, Warman ML. Preparation of PCR-quality mouse genomic DNA with hot sodium hydroxide and tris (HotSHOT). Biotechniques 2000;29: 54.

7. Luks VL, Kamitaki N, Vivero MP, et al. Lymphatic and other vascular malformative/overgrowth disorders are caused by somatic mutations in PIK3CA. J Pediatr 2015;166:1048-54.

8. Huang L, Nakayama H, Klagsbrun M, Klagsbrun M, Mulliken JB, Bischoff J. Glucose transporter 1-positive endothelial cells in infantile hemangioma exhibit features of facultative stem cells. Stem Cells 2015;33:133-45.

9. Samuels Y, Wuang Z, Bardelli A, et al. High frequency of mutations of the PIK3CA gene in human cancers. Science 2004;304:554.

10. Brown NK, Zhou Z, Zhang J, et al. Perivascular adipose tissue in vascular function and disease: a review of current research and animal models. Arterioscler Thromb Vasc Biol 2014;34:1621-30.

11. Couto JA, Huang L, Vivero MP, et al. Endothelial cells from capillary malformations are enriched for somatic GNAQ mutations. Plast Reconstr Surg 2016;137:77e-82e.

12. Kurek KC, Luks VL, Ayturk UM, et al. Somatic mosaic activating mutations in PIK3CA cause CLOVES syndrome. Am J Hum Genet. 20128:90:1108-5.

13. Couto JA, Vivero MP, Upton J, et al. Facial infiltrating lipomatosis contains somatic PIK3CA mutations in multiple tissues. Plast Reconstr Surg 2015;136(4 Suppl):72-3.

14. Pansky B. Germ Layer and Their Derivatives. Review of Medical Embryology. MacMillan Publishing: New York, New York, 1982: 62-63.

15. Larsen WJ. The Third WeekHuman Embryology. Churchill Livingstone: New York, New York, 1993: 47-63. 